\title{
Correction to: Euclidean Design Theory
}

\section{Correction to:}

\section{Sawa et al., Euclidean Design Theory, JSS Research Series in Statistics, https://doi.org/10.1007/978-981-13-8075-4}

In the original version of the book, the following belated corrections have been made.

In the following list, line $n$ means $n$-th line from the top and line $-n$ means $n$-th line from the bottom:

Page 4, line -7: Proof of Proposition 1.1(ii),

“ $\sum_{i, j} c_{i} c_{j} K_{i j}^{(1)} K_{i j}^{(2)}=\sum_{i, j} c_{i} c_{j}\left(\sum_{k} \lambda_{k} v_{k i} v_{k j}\right) \sum_{i, j} K_{i j}^{(2)}$,

has been replaced by

“ $\sum_{i, j} c_{i} c_{j} K_{i j}^{(1)} K_{i j}^{(2)}=\sum_{i, j} c_{i} c_{j}\left(\sum_{k} \lambda_{k} v_{k i} v_{k j}\right) K_{i j}^{(2)} "$.

Page 21, line 1: " $\int_{-1}^{1} \cdot d u / 2$ " has been replaced by " $\int_{0}^{1} \cdot d u$ ". 
Page 80 , line -7 has been changed as follows:

$$
\frac{1}{\left(1-u^{4}\right)\left(1-u^{6}\right) \cdots\left(1-u^{2 d-2}\right)\left(1-u^{d}\right)}=\left\{\begin{array}{cl}
1+2 u^{4}+u^{6}+3 u^{8}+\mathcal{O}\left(u^{10}\right), & \text { if } \mathrm{d}=4, \\
1+u^{4}+u^{5}+u^{6}+2 u^{8}+\mathcal{O}\left(u^{9}\right), & \text { if } \mathrm{d}=5, \\
1+u^{4}+2 u^{6}+2 u^{8}+\mathcal{O}\left(u^{10}\right), & \text { if } \mathrm{d}=6, \\
1+u^{4}+u^{6}+u^{7}+2 u^{8}+\mathcal{O}\left(u^{10}\right), & \text { if } \mathrm{d}=7, \\
1+u^{4}+u^{6}+3 u^{8}+\mathcal{O}\left(u^{10}\right), & \text { if } \mathrm{d}=8, \\
1+u^{4}+u^{6}+2 u^{8}+\mathcal{O}\left(u^{10}\right), & \text { if } \mathrm{d} \geq 9 .
\end{array}\right.
$$

Page 81, line 7 has been changed as follows:

$$
\operatorname{dim} \operatorname{Harm}_{8}\left(\mathbb{R}^{d}\right)^{D_{d}}= \begin{cases}3, & d=4,8 \\ 2, & d \geq 5, d \neq 8 .\end{cases}
$$

Page 81 , line -10 : “ $d \geq 4, d \neq 6$ " has been replaced by " $d \geq 4$ ".

Page 81, line -6: "the following polynomials $f_{8,1}, f_{8,2}, f_{8,3}$, respectively, as" has been replaced by "the following polynomials $f_{8,1}, f_{8,2}, f_{8,3}, f_{8,4}$, respectively, as".

Page 82, line 1: the following has been included:

For $d=8$

$$
f_{8,4}=x_{1} x_{2} x_{3} x_{4} x_{5} x_{6} x_{7} x_{8}
$$

Page 82, line -10 : "For $d \geq 4, d \neq 6$ " has been replaced by "For $d \geq 4$ ".

Page 83, line 5: the following has been included:

(iv) For $d=8$

$$
f_{8,4}\left(v_{1}\right)=\cdots=f_{8,4}\left(v_{6}\right)=0, \quad f_{8,4}\left(v_{7}\right)=-\frac{1}{4096}, \quad f_{8,4}\left(v_{8}\right)=\frac{1}{4096} .
$$

Page 99, line 8 has been changed as follows:

$$
\begin{aligned}
f_{8,2}\left(v_{A, s}\right)=\frac{1}{(A+s)^{4}} & \left\{A^{2} s+\frac{(s-1) s}{2}-\frac{6}{d-2}\left(A(s-1) s+\frac{A^{2}(s-1) s}{2}\right.\right. \\
& \left.\left.+\frac{(s-2)(s-1) s}{2}\right)+\frac{9 s(-3+4 A+s)(s-2)(s-1)}{2(d-3)(d-2)}\right\} .
\end{aligned}
$$

The correction chapters have been updated with these changes. 